\title{
Toric Calabi-Yau hypersurfaces fibered by weighted K3 hypersurfaces
}

\author{
Joshua P. Mullet
}

\begin{abstract}
In response to a question of Reid, we find all anti-canonical CalabiYau hypersurfaces $X$ in toric weighted $\mathbb{P}^{3}$-bundles over $\mathbb{P}^{1}$ where the general fiber of $X$ over $\mathbb{P}^{1}$ is a weighted K3 hypersurface. This gives a direct generalization of Reid's discovery of the 95 families of weighted K3 hypersurfaces in [18]. We also treat the case where $X$ is fibered over $\mathbb{P}^{2}$ with general fiber a genus one curve in a weighted projective plane.
\end{abstract}

\section{Introduction}

In light of the successes of the minimal model program in the classification of 3-folds, Corti and Reid promulgate in [2] a program of explicit birational study of 3-folds. Simply put, the interest is to obtain explicit equations for 3 -folds as hypersurfaces or complete intersections in simple varieties such as projective spaces or weighted projective spaces, and then focus study to these explicit examples, regarding them as birational models. An early example of such an explicit result is Reid's discovery of the 95 families of K3 hypersurfaces in weighted projective 3 -space (see [18]).

Miles Reid posed to the author the problem of classifying Calabi-Yau 3 -folds fibered over the projective line where the general fiber is one of the 95 weighted K3 hypersurfaces mentioned in the previous paragraph. Since the fibers are to be weighted hypersurfaces, a natural place to look for such 3 -folds is in weighted projective bundles. Furthermore, if we are interested in being explicit, we should look for these 3 -folds in some "simple" varieties. For us, those simple varieties are toric varieties. We are thus naturally led to the problem of finding K3-fibered Calabi-Yau 3-folds in toric weighted projective bundles over $\mathbb{P}^{1}$. In this paper, we find all such 3 -folds and thereby obtain a direct generalization of Reid's discovery of the 95 families of weighted K3 hypersurfaces.

The contents of this paper are as follows. In Section 2, we fix some definitions and notation regarding the theory of toric varieties that we use throughout the paper. In Section 3, we construct weighted projective bundles 
as toric varieties. Weighted projective bundles can also be obtained by taking Proj of a sheaf of weighted polynomial algebras. In Section 4 we describe the Proj construction and show that it agrees with the construction using toric varieties. In Section 5, we describe the intersection theory of weighted $\mathbb{P}^{1}$ bundles over $\mathbb{P}^{1}$ in terms of the well-known intersection theory on ordinary $\mathbb{P}^{1}$-bundles. We will need this description in the proof of Theorem 8.5. In Section 6 , we consider linear systems on toric varieties and, more specifically, on weighted projective spaces and bundles. Section 7 is a technical section in which we show that the condition of well-formedness (Definition 2.2), which is a condition imposed on hypersurfaces to make the adjunction formula work as expected, is automatic in the case of quasi-smooth hypersurfaces (Definition 2.1). In Section 8, we prove our main result, Theorem 8.5, in which we give necessary and sufficient conditions for a toric weighted projective bundle over $\mathbb{P}^{1}$ to admit a K3-fibered anti-canonical Calabi-Yau hypersurface, thus providing an answer to Reid's original question. Section 9 contains a statement of the analogous result for elliptically fibered Calabi-Yau 3-folds over $\mathbb{P}^{2}$ whose general fiber is a genus one curve in a weighted $\mathbb{P}^{3}$. The complete list of all our Calabi-Yau varieties is quite long, but the appendix contains a sampling of data, and we refer the reader to [17] for all the data and details of the calculation.

We should mention that Masanori Kobayashi considered the problem in the non-weighted case [14] and without the explicit use of toric varieties. Our work is inspired by his, and we use some of his notation in Section 6 .

\section{Acknowledgments}

I would like to thank my advisers, Dan Grayson and Sheldon Katz, for their attention and encouragement. I would also like to thank Miles Reid for suggesting the problem, Masanori Kobayashi for sharing unpublished work with me, Herb Clemens for his suggestions and Dario Sanchez Gomez for his corrections.

\section{Conventions and notation}

We work over $\mathbb{C}$, the field of complex numbers, and we follow standard conventions regarding the theory of toric varieties as described, for example, in [5] or [7]. If $X$ is a toric variety, we will denote its associated lattices as $N_{X}$ and $M_{X}:=\operatorname{Hom}_{\mathbb{Z}}\left(N_{X}, \mathbb{Z}\right)$, and if $N$ is a lattice we define $N_{\mathbb{R}}:=$ $N \otimes_{\mathbb{Z}} \mathbb{R}$. We always assume that our toric varieties have the property that 
all their maximal cones have dimension equal to the rank of the lattice used to define them.

We will need the notions of quasi-smoothness and well-formedness, which we define below for subvarieties of toric varieties. Let $X_{\Sigma}$ be a simplicial toric variety associated to a fan $\Sigma$, and let $S_{\Sigma}$ be its homogeneous coordinate ring with irrelevant ideal $B \subseteq S_{\Sigma}$ (see [3]). According to [3, Theorem 2.1], $X_{\Sigma}$ is isomorphic to the geometric quotient

$$
\left(\operatorname{Spec} S_{\Sigma}-Z(B)\right) / G
$$

where

$$
G=\operatorname{Hom}_{\mathbb{Z}}\left(A^{1} X_{\Sigma}, \mathbb{C}^{*}\right)
$$

Definition 2.1. Let

$$
\operatorname{Spec} S_{\Sigma}-Z(B) \stackrel{q}{\rightarrow} X_{\Sigma}
$$

be the quotient map. We say that a closed subvariety $Y \subseteq X_{\Sigma}$ is quasismooth if $q^{-1} Y$ is non-singular.

Definition 2.2. Let $X_{\Sigma}$ be a simplicial toric variety, and let $\operatorname{sing} X_{\Sigma}$ denote the singular locus of $X_{\Sigma}$. We say that a closed subvariety $Y \subseteq X_{\Sigma}$ is wellformed if the codimension of $\left(\operatorname{sing} X_{\Sigma}\right) \cap Y$ is at least two in $Y$.

Remark 2.3. If $Y \subseteq X_{\Sigma}$ is quasi-smooth, then $Y$ is an orbifold.

\section{Weighted projective bundles as toric varieties}

In this section, we consider the problem of constructing weighted projective bundles as toric varieties. We first recall how to construct the weighted projective space

$$
\mathbb{P}\left(a_{0}, \ldots, a_{n}\right)
$$

as a toric variety. 
Construction 3.1. Let $\left(a_{0}, \ldots, a_{n}\right)$ be a list of positive integers such that

$$
\operatorname{gcd}\left(a_{0}, \ldots, \widehat{a_{i}}, \ldots, a_{n}\right)=1
$$

where $\widehat{a_{i}}$ means that we omit $a_{i}$. Then form the weighted projective space

$$
\mathbb{P}\left(a_{0}, \ldots, a_{n}\right):=\operatorname{Proj} \mathbb{C}\left[X_{0}, \ldots, X_{n}\right]
$$

where $\operatorname{deg} X_{i}=a_{i}$. (See $[6,13]$ for generalities about weighted projective space.) As described in [7], we may write $\mathbb{P}\left(a_{0}, \ldots, a_{n}\right)$ as a toric variety as follows. Define the lattice $N$ via the following exact sequence:

$$
0 \longrightarrow \mathbb{Z} \frac{\left(\begin{array}{c}
a_{0} \\
\vdots \\
a_{n}
\end{array}\right)}{\longrightarrow} \mathbb{Z}^{n+1} \longrightarrow N \longrightarrow 0
$$

and define the fan $\Sigma \subseteq N_{\mathbb{R}}$ to consist of cones spanned by the images in $N_{\mathbb{R}}$ of proper subsets of the standard basis for $\mathbb{Z}^{n+1}$. Then

$$
\mathbb{P}\left(a_{0}, \ldots, a_{n}\right) \cong X_{\Sigma}
$$

Note that if we take $a_{i}=1$ for all $0 \leq i \leq n$, we obtain a description of (ordinary) projective space as a toric variety.

We now generalize the construction above to obtain weighted projective space bundles over $\mathbb{P}^{k}$.

Construction 3.2. Let $n$ and $k$ be positive integers, let $\left(a_{0}, \ldots, a_{n}\right)$ be a list of positive integers satisfying (3.1), and let $\left(d_{0}, \ldots, d_{n}\right)$ be an arbitrary list of integers. Define a lattice $N$ via the following exact sequence:

$$
0 \longrightarrow \mathbb{Z}^{2} \frac{}{\left(\begin{array}{cc}
a_{0} & -d_{0} \\
\vdots & \vdots \\
a_{n} & -d_{n} \\
0 & 1 \\
\vdots & \vdots \\
0 & 1
\end{array}\right)} \mathbb{Z}^{n+1} \oplus \mathbb{Z}^{k+1} \longrightarrow N \longrightarrow 0
$$

Now let $\left\{e_{0}, \ldots, e_{n}\right\}$ denote the standard basis for $\mathbb{Z}^{n+1}$, and let $\left\{f_{0}, \ldots, f_{k}\right\}$ denote the standard basis for $\mathbb{Z}^{k+1}$. We let $\Sigma \subseteq N_{\mathbb{R}}$ be the fan that consists 
of cones spanned by the images in $N_{\mathbb{R}}$ of proper subsets of the standard bases that contain neither all of the $e_{i}$ nor all of the $f_{i}$.

Proposition 3.3. Let $\Sigma$ be the fan defined in Construction 3.2, and let $X_{\Sigma}$ be the associated toric variety. Then $X_{\Sigma}$ is a locally trivial fibration over $\mathbb{P}^{k}$ with fiber $\mathbb{P}\left(a_{0}, \ldots, a_{n}\right)$.

Proof. Let $N_{X_{\Sigma}}$ be the lattice for $X_{\Sigma}$, let $N_{\mathbb{P}^{k}}$ be the lattice for $\mathbb{P}^{k}$, and let $N_{\mathbb{P}\left(a_{0}, \ldots, a_{n}\right)}$ be the lattice for $\mathbb{P}\left(a_{0}, \ldots, a_{n}\right)$; here we regard $\mathbb{P}^{k}$ and $\mathbb{P}\left(a_{0}, \ldots, a_{n}\right)$ as toric varieties according to Construction 3.1. We have the following commutative diagram whose rows and columns are exact.

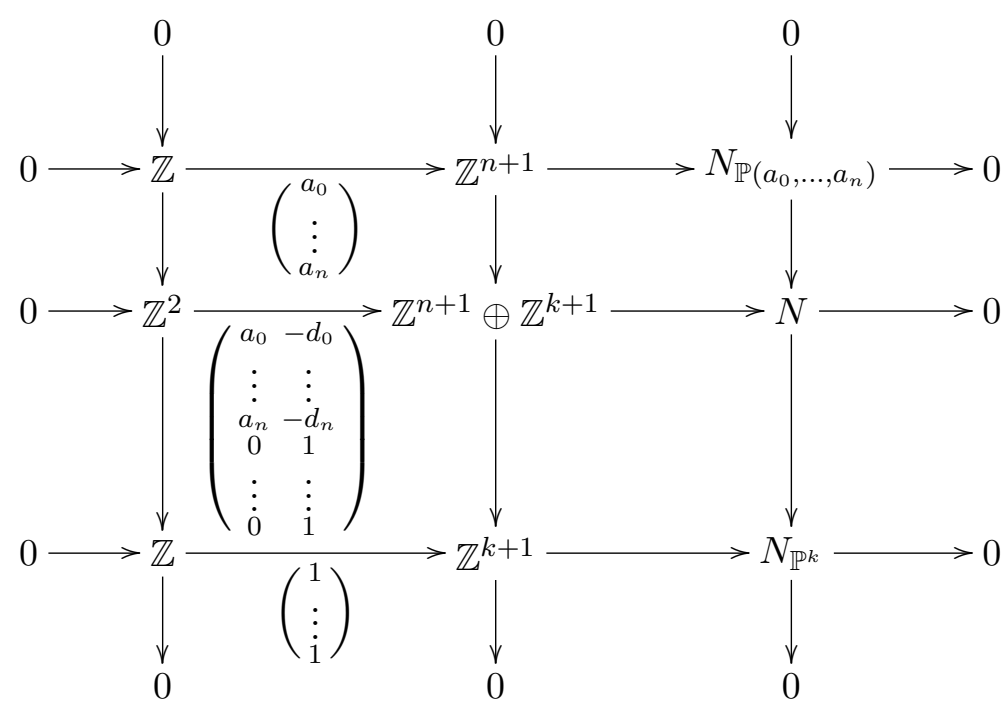

The right column of (3.4) gives the required maps of lattices. Furthermore, since the middle column is just inclusion of the first factor followed by projection onto the second factor, we can canonically extend the maps of lattices to maps of fans. The result now follows from [7, p. 41, Exercise].

Definition 3.4. We call the toric varieties from Construction 3.2 toric weighted projective bundles.

We conclude this section by finding the homogeneous coordinate rings and irrelevant ideals (in the sense of [3]) of toric weighted projective bundles. The description given in Construction 3.2 makes this particularly easy. 
Recall that for a toric variety $X$ there exists an exact sequence

$$
0 \longrightarrow M \longrightarrow \mathbb{Z}^{\Sigma(1)} \longrightarrow A^{1} X \longrightarrow 0,
$$

where $\Sigma(1)$ denotes the set of one-dimensional cones in the fan, and where $A^{1} X$ denotes the Chow group of codimension one cycles modulo rational equivalence. We begin with a proposition in which we compute the exact sequence (3.5) for toric weighted projective bundles.

Proposition 3.5. Let $\left(a_{0}, \ldots, a_{n}\right)$ be a sequence of positive integers satisfying (3.1), and let $\widetilde{\mathbb{P}}$ be a locally trivial toric fibration over $\mathbb{P}^{k}$ with fiber $\mathbb{P}\left(a_{0}, \ldots, a_{n}\right)$. There is an isomorphism of exact sequences:

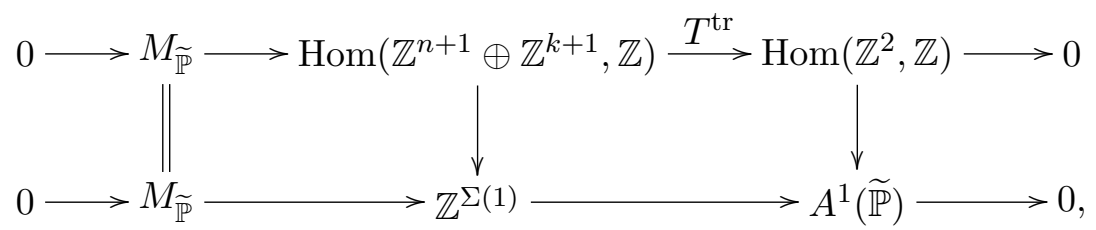

where $T$ is the matrix in Construction 3.2 and the bottom row is the standard exact sequence (3.5).

Proof. Since the rays of the fan for $\widetilde{\mathbb{P}}$ are given by the images of standard basis vectors under the cokernel of the map $T$, the result will follow immediately provided we show that the images of these vectors are primitive vectors in $N_{\widetilde{\mathbb{P}}}$. Let $\left(e_{0}, \ldots, e_{n}\right)$ be the standard basis for $\mathbb{Z}^{n+1}$ and let $\left(f_{0}, \ldots, f_{k}\right)$ be the standard basis for $\mathbb{Z}^{k+1}$. Without loss of generality, we consider the case in which the image of $e_{0}$ or $f_{0}$ is not primitive in $N_{\widetilde{\mathbb{P}}}$. Let

$$
r_{1}=\left(\begin{array}{c}
a_{0} \\
\vdots \\
a_{n} \\
0 \\
\vdots \\
0
\end{array}\right) \quad \text { and } \quad r_{2}=\left(\begin{array}{c}
-d_{0} \\
\vdots \\
-d_{n} \\
1 \\
\vdots \\
1
\end{array}\right)
$$

be the columns of the matrix $T$. Since the image of $e_{0}$ is not primitive in $N_{\mathbb{P}}$ or the image of $f_{0}$ is not, there exists a vector $v \in \mathbb{Z}^{n+1} \oplus \mathbb{Z}^{k+1}$ such that

$$
e_{0}+n v \in \operatorname{Span}\left\{r_{1}, r_{2}\right\} \quad \text { or } \quad f_{0}+n v \in \operatorname{Span}\left\{r_{1}, r_{2}\right\},
$$

for some integer $n$ such that $|n|>1$. 
In case the image of $e_{0}$ is not primitive in $N_{\widetilde{\mathbb{P}}}$ then

$$
e_{0}+n v=c_{1} r_{1}+c_{2} r_{2}
$$

for some integers $c_{1}$ and $c_{2}$. Note that $n$ does not divide $\operatorname{gcd}\left(c_{1}, c_{2}\right)$. If we consider the components of $n v$, we find that $n \mid c_{2}$ and hence $n \mid c_{1} a_{i}$ for all $i \neq 0$, and this contradicts (3.1). Thus $n=1$ and $v$ is primitive.

In case the image of $f_{0}$ is not primitive in $N_{\widetilde{\mathbb{P}}}$ then

$$
f_{0}+n v=c_{1} r_{1}+c_{2} r_{2}
$$

for some integers $c_{1}$ and $c_{2}$. Considering the components of $n v$ we find that $n \mid c_{2}$ and $n \mid\left(c_{2}-1\right)$, hence $n=1$, and again we have a contradiction.

We are now ready to write down the homogeneous coordinate ring and irrelevant ideal $B$ for a toric $\mathbb{P}\left(a_{0}, \ldots, a_{n}\right)$-bundle over $\mathbb{P}^{k}$.

Corollary 3.6. Let $\left(a_{0}, \ldots, a_{n}\right)$ be a sequence of positive integers satisfying (3.1), and let $\widetilde{\mathbb{P}}$ be a toric weighted projective over $\mathbb{P}^{k}$ with fiber $\mathbb{P}\left(a_{0}, \ldots, a_{n}\right)$. Then the homogeneous coordinate ring of $\widetilde{\mathbb{P}}$ is isomorphic to the $\mathbb{Z}^{2}$-graded polynomial ring

$$
\mathbb{C}\left[X_{0}, \ldots, X_{n}, S_{0}, \ldots, S_{k}\right]
$$

where

$$
\begin{aligned}
\operatorname{deg} X_{i} & =\left(a_{i},-d_{i}\right) \quad \text { and } \\
\operatorname{deg} S_{j} & =(0,1) .
\end{aligned}
$$

Furthermore, the irrelevant ideal $B \subseteq S_{X}$ is given by

$$
B=\left(X_{0} S_{0}, \ldots, X_{n} S_{0}, X_{0} S_{1}, \ldots, X_{n} S_{1}, \ldots, X_{0} S_{k}, \ldots, X_{n} S_{k}\right)
$$

Proof. This follows immediately from Proposition 3.5 and the construction in [3] of the homogeneous coordinate ring of a toric variety.

\section{Weighted projective bundles as Proj of weighted symmetric algebras}

In this section, we view weighted projective bundles over projective space as Proj of weighted symmetric algebras of sums of invertible sheaves and 
show that they are isomorphic to the toric varieties in Construction 3.2. For convenience, we adopt the following definition.

Definition 4.1. Let $X$ be a scheme, and let $\left(a_{0}, \ldots, a_{n}\right)$ be a sequence of positive integers. We define a weighted locally free sheaf with weights $\left(a_{0}, \ldots, a_{n}\right)$ to be a locally free sheaf of $\mathcal{O}_{X}$-modules $\mathcal{E}$ together with an ordered decomposition $\mathcal{E} \cong \mathcal{E}_{0} \oplus \cdots \oplus \mathcal{E}_{n}$ such that $\mathcal{E}_{i}$ is an invertible sheaf and such that the direct sum is to be interpreted as a graded sheaf with $\mathcal{E}_{i}$ placed in degree $a_{i}$ for $0 \leq i \leq n$. The weights will be used in Definition 4.3 when we form symmetric algebras of weighted locally free sheaves.

Remark 4.2. It certainly is possible not to require that the locally free sheaves $\mathcal{E}_{i}$ in the decomposition in Definition 4.1 have rank one, but we will restrict to this case as this is case that relates to toric varieties.

Definition 4.3. Let $X$ be a scheme. Given a weighted locally free sheaf $\mathcal{E}$ with weights $\left(a_{0}, \ldots, a_{n}\right)$ let $\mathcal{S}$ denote the weighted symmetric algebra of $\mathcal{E}$ where we declare that $\mathcal{E}_{i}$ have homogeneous degree $a_{i}$ in $\mathcal{S}$. We define the weighted projective bundle associated to $\mathcal{E}$ to be the $X$-scheme

$$
\widetilde{\mathbb{P}}(\mathcal{E}):=\operatorname{Proj} \mathcal{S} \longrightarrow X
$$

We have the following two basic lemmas for weighted projective bundles.

Lemma 4.4. Let $X$ be a variety over $\mathbb{C}$, and let $\mathcal{E}$ be a weighted locally free sheaf on $X$ with weights $\left(a_{0}, \ldots, a_{n}\right)$. Then the weighted projective bundle $\widetilde{\mathbb{P}}(\mathcal{E})$ is a locally trivial fiber bundle over $X$ with fiber the weighted projective space $\mathbb{P}\left(a_{0}, \ldots, a_{n}\right)$.

Proof. This follows from the construction of Proj of a sheaf of graded algebras as described, for example, in [11, Section 3].

Lemma 4.5. Let $X$ be a scheme, let $\mathcal{E}$ be a weighted locally free sheaf on $X$ with weights $\left(a_{0}, \ldots, a_{n}\right)$, and let $\mathcal{L}$ be an invertible sheaf of $\mathcal{O}_{X}$-modules. Then there is a canonical $X$-isomorphism

$$
\widetilde{\mathbb{P}}(\mathcal{E}) \cong \widetilde{\mathbb{P}}\left(\bigoplus_{i=0}^{n} \mathcal{E}_{i} \otimes \mathcal{L}^{\otimes a_{i}}\right)
$$

Proof. This is just the application to our special case of [11, Proposition 3.1.8 (iii)]. 
We also state the relative version of [13, Lemma 5.7].

Lemma 4.6. Let $\left(a_{0}, \ldots, a_{n}\right)$ be a sequence of positive integers having no common factor, let $X$ be a scheme, and let $\mathcal{E}=\bigoplus_{i=0}^{n} \mathcal{E}_{i}$ be a weighted locally free sheaf of $\mathcal{O}_{X}$-modules with weights $\left(a_{0}, \ldots, a_{n}\right)$. Let $q$ be a positive integer such that $q \mid a_{i}$ for $i>0$, and define a new weighted locally free sheaf $\mathcal{E}^{\prime}$ with weights $\left(a_{0}, \frac{a_{1}}{q}, \ldots, \frac{a_{n}}{q}\right)$ via

$$
\mathcal{E}^{\prime}:=\mathcal{E}_{0}^{\otimes q} \oplus \bigoplus_{i=1}^{n} \mathcal{E}_{i}
$$

Then $\widetilde{\mathbb{P}}(\mathcal{E}) \cong \widetilde{\mathbb{P}}\left(\mathcal{E}^{\prime}\right)$.

We conclude this section by relating the two notions of weighted projective bundles that we have described. We point out that Proposition 4.7 is a generalization of the exercise [7, Exercise p. 42].

Proposition 4.7. Let $\left(a_{0}, \ldots, a_{n}\right)$ be a sequence of positive integers satisfying (3.1), and let $d_{0}, \ldots, d_{n}$ be integers. Let $\mathcal{E}$ denote the weighted locally free sheaf

$$
\mathcal{E}:=\mathcal{O}\left(d_{0}\right) \oplus \cdots \oplus \mathcal{O}\left(d_{n}\right)
$$

on $\mathbb{P}^{k}$ with weights $\left(a_{0}, \ldots, a_{n}\right)$. Then $\widetilde{\mathbb{P}}(\mathcal{E})$ is isomorphic to $\widetilde{\mathbb{P}}$, the toric weighted projective bundle from Construction 3.2.

Proof. We first fix some notation. Write $\mathbb{P}^{k}=\operatorname{Proj} \mathbb{C}\left[S_{0}, \ldots S_{k}\right]$ and let $U_{i}=$ Spec $\mathbb{C}\left[S_{0} / S_{i}, \ldots, S_{k} / S_{i}\right]$. Let $\left\{X_{i}\right\}_{i=0}^{n}$ be global coordinates on $\widetilde{\mathbb{P}}(\mathcal{E})$; this means that

$$
\left.\widetilde{\mathbb{P}}(\mathcal{E})\right|_{U_{i}} \cong \operatorname{Proj}\left(\mathbb{C}\left[\frac{S_{0}}{S_{i}}, \ldots, \frac{S_{k}}{S_{i}}\right]\left[S_{i}^{d_{0}} X_{0}, \ldots, S_{i}^{d_{n}} X_{n}\right]\right)
$$

where $\operatorname{deg} S_{i}^{d_{j}} X_{j}=a_{j}$. We find for $0 \leq i \leq k$ that $\left.\widetilde{\mathbb{P}}(\mathcal{E})\right|_{U_{i}}$ is isomorphic to the toric variety $\mathbb{A}^{k} \times \mathbb{P}\left(a_{0}, \ldots, a_{n}\right)$. Let $U:=\bigcap_{i} U_{i}$. Distinguishing $S_{0}$, we find

$$
\left.\widetilde{\mathbb{P}}(\mathcal{E})\right|_{U} \cong \operatorname{Proj}\left(\mathbb{C}\left[\left(\frac{S_{1}}{S_{0}}\right)^{ \pm 1}, \ldots,\left(\frac{S_{k}}{S_{0}}\right)^{ \pm 1}\right]\left[S_{0}^{d_{0}} X_{0}, \ldots, S_{0}^{d_{n}} X_{n}\right]\right)
$$


where $\operatorname{deg} S_{0}^{d_{j}} X_{j}=a_{j}$. Distinguishing $S_{0}$ amounts to choosing a basis for the torus $\mathbb{T}^{k}$ in $\mathbb{P}^{k}$ and thereby identifying the lattice $M_{\mathbb{P}^{k}}$ with $\mathbb{Z}^{k}$. Similarly, the coordinates $S_{0}^{d_{i}} X_{i}$ correspond to a basis for $\mathbb{Z}^{n+1}$ in the context of the exact sequence (3.2). Therefore, the inclusion

$$
\left.\left.\widetilde{\mathbb{P}}(\mathcal{E})\right|_{U} \longrightarrow \widetilde{\mathbb{P}}(\mathcal{E})\right|_{U_{i}}
$$

induces an isomorphism

$$
M_{U_{i}} \oplus \mathbb{Z}^{n+1} \stackrel{\phi_{i}}{\longrightarrow} M_{\mathbb{T}^{k}} \oplus \mathbb{Z}^{n+1},
$$

which is the identity if $i=0$ and if $i>0$ is given by the matrix

$$
\left(\begin{array}{ccccccccccc}
0 & 1 & \ldots & 0 & 0 & \ldots & 0 & 0 & 0 & \ldots & 0 \\
\vdots & \vdots & \ddots & \vdots & \vdots & \ddots & \vdots & \vdots & \vdots & \ddots & \vdots \\
0 & 0 & \ldots & 1 & 0 & \ldots & 0 & 0 & 0 & \ldots & 0 \\
-1 & -1 & \ldots & -1 & -1 & \ldots & -1 & d_{0} & d_{1} & \ldots & d_{n} \\
0 & 0 & \ldots & 0 & 1 & \ldots & 0 & 0 & 0 & \ldots & 0 \\
\vdots & \vdots & \ddots & \vdots & \vdots & \ddots & \vdots & \vdots & \vdots & \ddots & \vdots \\
0 & 0 & \ldots & 0 & 0 & \ldots & 1 & 0 & 0 & \ldots & 0 \\
0 & 0 & \ldots & 0 & 0 & \ldots & 0 & 1 & 0 & \ldots & 0 \\
0 & 0 & \ldots & 0 & 0 & \ldots & 0 & 0 & 1 & \ldots & 0 \\
\vdots & \vdots & \ddots & \vdots & \vdots & \ddots & \vdots & \vdots & \vdots & \ddots & \vdots \\
0 & 0 & \ldots & 0 & 0 & \ldots & 0 & 0 & 0 & \ldots & 1
\end{array}\right)
$$

where

$$
\left(\begin{array}{lllllllllll}
-1 & -1 & \cdots & -1 & -1 & \cdots & -1 & d_{0} & d_{1} & \cdots & d_{n}
\end{array}\right)
$$

is the $i$ th row. Taking the transpose of $\phi_{i}$ and composing with the map in (3.2) we obtain isomorphisms

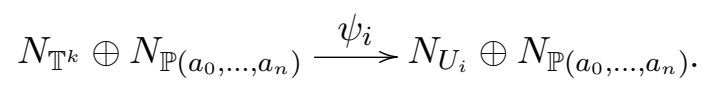

We put

$$
N_{\widetilde{\mathbb{P}}(\mathcal{E})}:=N_{\mathbb{T}^{k}} \oplus N_{\mathbb{P}\left(a_{0}, \ldots, a_{n}\right)},
$$

and the rays of the fan are the inverse images under the isomorphisms $\psi_{i}$ of the rays of the fans for $U_{i} \times \mathbb{P}\left(a_{0}, \ldots, a_{n}\right)$. Observe that the inverse of the 
matrix (4.1) is given by

$$
\left(\begin{array}{cccccccccccc}
-1 & -1 & \ldots & -1 & -1 & -1 & \ldots & -1 & d_{0} & d_{1} & \ldots & d_{n} \\
1 & 0 & \ldots & 0 & 0 & 0 & \ldots & 0 & 0 & 0 & \ldots & 0 \\
0 & 1 & \ldots & 0 & 0 & 0 & \ldots & 0 & 0 & 0 & \ldots & 0 \\
\vdots & \vdots & \ddots & \vdots & \vdots & \vdots & \ddots & \vdots & \vdots & \vdots & \ddots & \vdots \\
0 & 0 & \ldots & 1 & 0 & 0 & \ldots & 0 & 0 & 0 & \ldots & 0 \\
0 & 0 & \ldots & 0 & 0 & 1 & \ldots & 0 & 0 & 0 & \ldots & 0 \\
\vdots & \vdots & \ddots & \vdots & \vdots & \vdots & \ddots & \vdots & \vdots & \vdots & \ddots & \vdots \\
0 & 0 & \ldots & 0 & 0 & 0 & \ldots & 1 & 0 & 0 & \ldots & 0 \\
0 & 0 & \ldots & 0 & 0 & 0 & \ldots & 0 & 1 & 0 & \ldots & 0 \\
0 & 0 & \ldots & 0 & 0 & 0 & \ldots & 0 & 0 & 1 & \ldots & 0 \\
\vdots & \vdots & \ddots & \vdots & \vdots & \vdots & \ddots & \vdots & \vdots & \vdots & \ddots & \vdots \\
0 & 0 & \ldots & 0 & 0 & 0 & \ldots & 0 & 0 & 0 & \ldots & 1
\end{array}\right)
$$

where the $i$ th column is the column whose only nonzero entry is -1 . Therefore the set of rays is the set of images in $N_{\widetilde{\mathbb{P}}(\mathcal{E})}$ of the set of row vectors of the matrices (4.2) for all $i$. They are given by the row vectors (as opposed to the column vectors) because the matrix for the map $\psi_{i}$ is the transpose of the matrix (4.2). It is now straightforward to check the relations among this set of row vectors and verify that the fan for $\widetilde{\mathbb{P}}(\mathcal{E})$ is isomorphic to the fan described in Construction 3.2.

\section{Intersection numbers on weighted $\mathbb{P}^{1}$-bundles over $\mathbb{P}^{1}$}

Let $a_{0}$ and $a_{1}$ be integers that are relatively prime. In this section, we consider divisors on $\mathbb{P}\left(a_{0}, a_{1}\right)$-bundles over $\mathbb{P}^{1}$. By Lemma 4.6 , such bundles are isomorphic to non-weighted $\mathbb{P}^{1}$-bundles over $\mathbb{P}^{1}$, and we check how divisors transform under this isomorphism so that we may apply the formula (5.2) below to obtain intersection numbers on $\mathbb{P}\left(a_{0}, a_{1}\right)$. In order to fix terminology and notation, we recall below the intersection theory of $\mathbb{P}^{1}$-bundles over $\mathbb{P}^{1}$. See [8] for details.

Example 5.1. Let $a$ and $b$ be integers, and form the locally free sheaf

$$
\mathcal{E}:=\mathcal{O}_{\mathbb{P}^{1}}(a) \oplus \mathcal{O}_{\mathbb{P}^{1}}(b)
$$

over $\mathbb{P}^{1}$. Let $H \in A^{1} \mathbb{P}(\mathcal{E})$ be the class of a fiber over $\mathbb{P}^{1}$, and let $L \in A^{1} \mathbb{P}(\mathcal{E})$ be the divisor class corresponding to $\mathcal{O}_{\mathbb{P}(\mathcal{E})}(1)$. Then the Chow ring of $\mathbb{P}(\mathcal{E})$ 
is given by

$$
A^{*} \mathbb{P}(\mathcal{E}) \cong \frac{\mathbb{Z}[H, L]}{\left(H^{2}, L^{2}-(a+b) H L\right)}
$$

Let $D_{1}$ be a divisor of type $\left(d_{1}, e_{1}\right)$, that is $D_{1} \sim d_{1} L+e_{1} H$, and let $D_{2}$ be a divisor of type $\left(d_{2}, e_{2}\right)$. Then we find from (5.1), that the intersection number $D_{1} \cdot D_{2}$ is given by

$$
D_{1} \cdot D_{2}=\operatorname{deg}\left(D_{1} D_{2}\right)=(a+b) d_{1} d_{2}+d_{1} e_{2}+d_{2} e_{1}
$$

The calculation in (5.2) uses the fact that the cycle class $H L$ is the class of a point in $A^{2} \mathbb{P}(\mathcal{E})$.

Let $d_{0}$ and $d_{1}$ be integers, and let $\mathcal{E}:=\mathcal{O}_{\mathbb{P}^{1}}\left(d_{0}\right) \oplus \mathcal{O}_{\mathbb{P}^{1}}\left(d_{1}\right)$ be a weighted locally free sheaf with relatively prime weights $\left(a_{0}, a_{1}\right)$. Let $X_{0}$ and $X_{1}$ be global coordinates on $\widetilde{\mathbb{P}}(\mathcal{E})$ as in the proof of Proposition 4.7 , and let $S_{0}$ and $S_{1}$ be coordinates on the base $\mathbb{P}^{1}$. Each coordinate $X_{i}$ defines an effective divisor $Z\left(X_{i}\right)$ on $\widetilde{\mathbb{P}}(\mathcal{E})$ by taking Proj of the surjective map of weighted symmetric algebras induced by the surjective map of sheaves

$$
\mathcal{E} \longrightarrow \mathcal{E} / \mathcal{O}_{\mathbb{P}^{1}}\left(d_{i}\right)
$$

The coordinates $S_{i}$ give divisors that are the pullbacks of divisors from the base $\mathbb{P}^{1}$. From Lemma 4.6 we know that

$$
\widetilde{\mathbb{P}}(\mathcal{E}) \cong \mathbb{P}\left(\mathcal{E}^{\prime}\right)
$$

where $\mathcal{E}^{\prime}=\mathcal{O}_{\mathbb{P}^{1}}\left(a_{1} d_{0}\right) \oplus \mathcal{O}_{\mathbb{P}^{1}}\left(a_{0} d_{1}\right)$. The next lemma shows how to realize a divisor given by global coordinates on $\widetilde{\mathbb{P}}(\mathcal{E})$ as a divisor given by global coordinates on $\mathbb{P}(\mathcal{E})$. For any real number $r$, the symbol $\lceil r\rceil$ denotes the smallest integer greater than or equal to $r$.

Lemma 5.2. Let $\mathcal{E}=\mathcal{O}_{\mathbb{P}^{1}}\left(d_{0}\right) \oplus \mathcal{O}_{\mathbb{P}^{1}}\left(d_{1}\right)$ be a weighted locally free sheaf with relatively prime weights $\left(a_{0}, a_{1}\right)$. Let $D=Z\left(S_{0}^{e_{0}} S_{1}^{e_{1}} X_{0}^{f_{0}} X_{1}^{f_{1}}\right)$ be an effective divisor on $\widetilde{\mathbb{P}}(\mathcal{E})$ defined by global coordinates. Let $D^{\prime}$ be the corresponding divisor on the isomorphic variety $\mathbb{P}\left(\mathcal{E}^{\prime}\right)$ where

$$
\mathcal{E}^{\prime}=\mathcal{O}_{\mathbb{P}^{1}}\left(a_{1} d_{0}\right) \oplus \mathcal{O}_{\mathbb{P}^{1}}\left(a_{0} d_{1}\right)
$$


Then $D^{\prime}=Z\left(S_{0}^{e_{0}} S_{1}^{e_{1}} X_{0}^{f_{0}^{\prime}} X_{1}^{f_{1}^{\prime}}\right)$ where for $i=0$ or $i=1$ and $j \neq i$ the integer $f_{i}^{\prime}$ is given by

$$
f_{i}^{\prime}=\left\lceil\frac{f_{i}}{a_{j}}\right\rceil
$$

Furthermore, the divisor $D^{\prime}$ has type

$$
\left(\left\lceil\frac{f_{0}}{a_{1}}\right\rceil+\left\lceil\frac{f_{1}}{a_{0}}\right\rceil, e_{0}+e_{1}-\left\lceil\frac{f_{0}}{a_{1}}\right\rceil a_{1} d_{0}-\left\lceil\frac{f_{1}}{a_{0}}\right\rceil a_{0} d_{1}\right) .
$$

Proof. The isomorphism of Lemma 4.6 can be realized by taking Proj of the degree $a_{0} a_{1}$ inclusion of sheaves of graded algebras

$$
\operatorname{Sym}_{\mathcal{O}_{\mathbb{P} 1}} \mathcal{E}^{\prime} \stackrel{h}{\longrightarrow} \widetilde{\operatorname{Sym}}_{\mathcal{O}_{\mathbb{P} 1}} \mathcal{E}
$$

where we have decorated the second "Sym" to emphasize that it is a weighted symmetric algebra. The divisor $D$ is given by a subsheaf of graded ideals

$$
\mathcal{I}_{0} \cdot \mathcal{J} \subseteq{\widetilde{\operatorname{Sym}_{\mathcal{O}_{\mathbb{1}}}}}_{\mathcal{E}}
$$

where $\mathcal{I}_{0}$ comes from the $S_{i}$ and is generated in degree 0 , and where the degree $d$ part of $\mathcal{J}$ is given by

$$
\mathcal{J}_{d}=\bigoplus_{\substack{k, l \geq 0, a_{0}\left(f_{0}+k\right)+\bar{a}_{1}\left(f_{1}+l\right)=d}} \mathcal{O}_{\mathbb{P}^{1}}\left(\left(f_{0}+k\right) d_{0}+\left(f_{1}+l\right) d_{1}\right) \cdot X_{0}^{f_{0}+k} X_{1}^{f_{1}+l} .
$$

Now, the divisor $D^{\prime}$ is defined by the sheaf of graded ideals

$$
h^{-1}\left(\mathcal{I}_{0} \cdot \mathcal{J}\right) \subseteq \operatorname{Sym}_{\mathcal{O}_{\mathbb{P} 1}} \mathcal{E}^{\prime}
$$

and the first assertion follows easily from the description of $\mathcal{J}_{d}$ above and the fact that $a_{0}$ and $a_{1}$ are relatively prime.

The second assertion follows from the fact that on any projective bundle

$$
\mathbb{P}\left(\mathcal{O}_{\mathbb{P}^{1}}\left(d_{0}\right) \oplus \mathcal{O}_{\mathbb{P}^{1}}\left(d_{1}\right)\right),
$$

the global coordinate $X_{i}$ defines a divisor of type $\left(1,-d_{i}\right)$, and the global coordinate $S_{j}$ defines a divisor of type $(0,1)$. This may be checked directly, and it also follows from our description of the bundle as a toric variety. 


\section{Linear systems}

Before we address the question of finding anti-canonical hypersurfaces in weighted projective bundles, we give some results regarding linear systems that we will need later. These results are generalizations of the standard results for linear systems on non-singular varieties as discussed, for example, in $[12]$ or $[10]$.

Let $X$ be a normal, projective variety, and let $\mathcal{L}$ be a coherent sheaf of $\mathcal{O}_{X}$-modules. Recall that $\mathcal{L}$ is reflexive if the canonical map

$$
\mathcal{L} \longrightarrow \mathcal{L}^{* *}
$$

is an isomorphism, where we define

$$
\mathcal{L}^{*}:=\mathcal{H}^{\circ o m_{\mathcal{O}_{X}}}\left(\mathcal{L}, \mathcal{O}_{X}\right) .
$$

Furthermore, if $\mathcal{L}$ is a reflexive subsheaf of the function field $k(X)$, then we say that $\mathcal{L}$ is divisorial. See [18] for details about divisorial sheaves. The main point is that to any Weil divisor $D$ on $X$, we may associate a divisorial sheaf $\mathcal{O}_{X}(D)$ and this assignment induces a one-to-one correspondence between the set of Weil divisors on $X$ modulo linear equivalence and the set of isomorphism classes of divisorial sheaves of $\mathcal{O}_{X}$-modules. The following fact is straightforward to check.

Lemma 6.1. Let $D$ be a Weil divisor on $X$. The set of effective divisors that are linearly equivalent to $D$ is in one-to-one correspondence with the set of closed points of the projective space

$$
\mathbb{P} H^{0}\left(X, \mathcal{O}_{X}(D)\right) .
$$

Definition 6.2. Let $X$ be a normal projective variety, and let $D$ be a Weil divisor on $X$. The complete linear system, denoted $|D|$, is the set of all effective Weil divisors that are linearly equivalent to $D$. A linear system is a linear subspace of a complete linear system, where we regard the complete linear system as having the structure of a projective space guaranteed by Lemma 6.1.

Definition 6.3. The base locus of a linear system $L$ is the set-theoretic intersection of the supports of the members of $L$.

Definition 6.4. Let $L \subseteq|D|$ be a linear system for some Weil divisor $D$ on $X$. We denote by $V_{L}$ the associated linear subspace of $H^{0}\left(X, \mathcal{O}_{X}(D)\right)$. 
Now suppose that $X_{\Sigma}$ is a simplicial toric variety associated to some fan $\Sigma \subseteq N_{\mathbb{R}}$ for some lattice $N$. Let $S$ be the homogeneous coordinate ring of $X_{\Sigma}$. Recall that $S$ is an $A^{1} X_{\Sigma}$-graded polynomial ring. Let $D$ be a Weil divisor on $X$. From [3, Proposition 1.1] we find that

$$
H^{0}\left(X_{\Sigma}, \mathcal{O}_{X_{\Sigma}}(D)\right) \cong S_{[D]},
$$

where $[D]$ denotes the class of the divisor $D$ in $A^{1}\left(X_{\Sigma}\right)$, and $S_{[D]}$ denotes the degree $[D]$ part of the graded ring $S$. One easily checks that the zero scheme (see [3]) of a homogeneous form $F \in S_{[D]}$ is a divisor on $X$ in the class of $D$ and, hence, that linear systems may be described as linear subspaces of $S_{[D]}$. We illustrate the case for weighted projective bundles in the following example.

Example 6.5. Any complete linear system $|D|$ on $\widetilde{\mathbb{P}}(\mathcal{E})$ may be written as the zero locus of forms

$$
\sum \phi_{e_{0} \cdots e_{n}}\left(S_{0}, \ldots, S_{k}\right) X_{0}^{e_{0}} \ldots X_{n}^{e_{n}} \subseteq S_{[D]}
$$

where the exponents $e_{i}$ satisfy $\sum a_{i} e_{i}=C$ for some integer $C$, and $\phi_{e_{0} \cdots e_{n}}$ is any form in $H^{0}\left(\mathbb{P}^{k}, \mathcal{O}(\eta)\right)$ for some integer-valued function $\eta$ that depends on the exponents $e_{i}$. In case $|D|=-K$, the function $\eta$ is given by

$$
\eta\left(e_{0}, \ldots, e_{n}\right):=\sum d_{i}\left(e_{i}-1\right)+k+1
$$

We now check that the property of a linear system on a toric variety having a quasi-smooth member is an open condition.

Lemma 6.6. Let $X$ be a simplicial toric variety that is proper over $\mathbb{C}$, and let $L$ be a non-empty linear system on $X$. Then there exists a quasi-smooth member of $L$ if and only if the general member of $L$ is quasi-smooth.

Proof. One direction is clear. Since $L$ is non-empty and the general member is quasi-smooth, it must be the case that $L$ contains a quasi-smooth member.

We now establish the converse. Assume that $L$ contains a quasi-smooth member. Consider the closed incidence subvariety $Z_{\text {sing }} \subseteq L \times X$ given by

$$
Z_{\text {sing }}:=\{[D] \times p \mid p \in \operatorname{sing} D\}
$$

where $\operatorname{sing} D$ denotes the locus where $D$ fails to be quasi-smooth. Let $\pi_{L}$ be the projection of $Z_{\text {sing }}$ onto $L$. The result now follows from the properness of $\pi_{L}$. 
One of the main tools in the analysis of linear systems is Bertini's Theorem. We check that a version of Bertini's Theorem holds that addresses the quasi-smoothness of members of linear systems on toric varieties.

Proposition 6.7. Let $X$ be a simplicial toric variety that is proper over $\mathbb{C}$, and let $L$ be a linear system on $X$. Then the general member of $L$ is quasi-smooth away from the base locus of $L$.

Proof. Let $S$ be the homogeneous coordinate ring of $X$, and let

$$
\operatorname{Spec} S-Z(B) \stackrel{q}{\longrightarrow} X
$$

be the quotient map (see Section 2). Since quasi-smoothness of a divisor on $X$ is determined by the non-singularity of a hypersurface on $\operatorname{Spec} S-Z(B)$, the argument given in the proof of Bertini's Theorem in [10, p. 137] applies in our case to establish the proposition.

It will be convenient to have the notion of the restriction of a linear system to the fiber of a weighted projective bundle.

Definition 6.8. Let $X$ be a simplicial toric variety, let $j: Y \hookrightarrow X$ be a well-formed quasi-smooth subvariety, and let $L \subseteq|D|$ be a linear system on $X$ for some Weil divisor $D$. Since $Y$ is well-formed, the sheaf $j^{*} \mathcal{O}_{X}(D)$ is divisorial. We define the restricted linear system, denoted $\left.L\right|_{Y}$ to be the linear system associated to the image of $V_{L}$ under the natural $\mathbb{C}$-linear map

$$
H^{0}\left(X, \mathcal{O}_{X}(D)\right) \longrightarrow H^{0}\left(Y, j^{*} \mathcal{O}_{X}(D)\right)
$$

Remark 6.9. Let $|D|$ be a complete linear system on a weighted projective space bundle, and let $F \cong \mathbb{P}\left(a_{0}, \ldots, a_{n}\right)$ be a fiber of the bundle. It follows easily from the description given in Example 6.5 that the restricted linear system $\mid D \|_{F}$ is independent of the choice of fiber $F$ when regarded as a linear system on $\mathbb{P}\left(a_{0}, \ldots, a_{n}\right)$.

Proposition 6.10. Let $|D|$ be a complete linear system on $\widetilde{\mathbb{P}}(\mathcal{E})$ over $\mathbb{P}^{k}$ for some divisor $|D|$, and let $F \cong \mathbb{P}\left(a_{0}, \ldots, a_{n}\right)$ be a fiber. If the general member of $|D|$ is quasi-smooth, then so is the general member of the restricted linear system $L:=\mid D \|_{F}$.

Proof. Suppose, to the contrary, that every member of $L$ fails to be quasismooth. We will show that the same is true for $|D|$. Let $G$ be the equation 
for a member of $|D|$. Restricting $G$ to any fiber, the assumption that every member of $L$ fails to be quasi-smooth implies that the system of equations

$$
G=\frac{\partial G}{\partial X_{0}}=\cdots=\frac{\partial G}{\partial X_{n}}=0
$$

has a non-trivial solution $P$. Furthermore, by Proposition 6.7, we may assume that $\left(X_{0}(P), \ldots, X_{n}(P)\right) \in \operatorname{Bs} L$. We claim that $P$ is also a solution to the system of equations

$$
\frac{\partial G}{\partial S_{0}}=\cdots=\frac{\partial G}{\partial S_{k}}=0 .
$$

Indeed, restricting the equations above to the fiber $F_{P}$ that contains $P$ yields a system of $(k+1)$ equations, each of which defines a member of $L$. But $\left.P\right|_{F_{P}} \in \operatorname{Bs} L$ by assumption, and the claim follows.

To conclude this section we state a result from [13] that addresses the problem of determining whether a linear system on $\mathbb{P}\left(a_{0}, \ldots, a_{3}\right)$ has a quasismooth member. In [13] the result is stated only for complete linear systems, but the result is a corollary of [13, 8.1 Theorem] whose proof applies more generally to linear systems whose associated vector space of homogeneous forms is generated by monomials.

Definition 6.11. Let $L$ be a linear system on weighted projective space. If the associated vector space of forms is generated by monomials, then we say that $L$ is a monomial linear system.

Proposition 6.12 ([13, 8.5 Corollary $])$. Let $\left(a_{0}, \ldots, a_{3}\right)$ be a sequence of positive integers satisfying (3.1), and let $L$ be a monomial linear system on $\mathbb{P}\left(a_{0}, \ldots, a_{3}\right)$ of degree $d$ where $d>a_{i}$ for all $0 \leq i \leq 3$. Then the general member of $L$ is quasi-smooth if and only if the following conditions hold.

(1) For all $0 \leq i \leq 3$ there exists a monomial in $V_{L}$ that does not involve $X_{i}$.

(2) For all $0 \leq i \leq 3$ there exists a monomial in $V_{L}$ of the form $X_{i}^{p} X_{e_{i}}$ for some $0 \leq e_{i} \leq 3$.

(3) For all $0 \leq i<j \leq 3$

(a) there exists a monomial in $V_{L}$ of the form $X_{i}^{p} X_{j}^{q}$ or

(b) there exist monomials in $V_{L}$ of the form $X_{i}^{p_{1}} X_{j}^{q_{1}} X_{e_{1}}$ and $X_{i}^{p_{2}} X_{j}^{q_{2}}$ $X_{e_{2}}$ for distinct $e_{i}$. 


\section{Well-formedness and anti-canonical hypersurfaces}

This section is concerned with the condition of well-formedness as given in Definition 2.2. In Section 8, we will apply the adjunction formula to a quasismooth anti-canonical hypersurface in a weighted projective bundle in order to show that it is a Calabi-Yau variety. For the adjunction formula to work as expected, we will need to know that the hypersurface in question is wellformed. As we now establish, it turns out that well-formedness is automatic for quasi-smooth anti-canonical hypersurfaces. In [13] Iano-Fletcher considered the problem of well-formedness of hypersurfaces in weighted projective space, and we take up this case sfirst.

In order to obtain information about the well-formedness of hypersurfaces in weighted projective space, we need to understand the singular locus of weighted projective space. All the singularities are finite cyclic quotient singularities, and we recall notation for such singularities below.

Definition 7.1. Let $a_{1}, \ldots, a_{n}$ be integers, let $r$ be a positive integer, and let $x_{1}, \ldots, x_{n}$ be coordinates on $\mathbb{A}^{n}$. Let $\mathbb{Z}_{r}$ act on $\mathbb{A}^{n}$ via

$$
x_{i} \mapsto \varepsilon^{a_{i}} x_{i} \text { for all } i,
$$

where $\varepsilon$ is a fixed primitive $r$ th root of unity. Let $X$ be an algebraic variety over $\mathbb{C}$. A singularity $Q \in X$ is a (quotient) singularity of type $\frac{1}{r}\left(a_{1}, \ldots, a_{n}\right)$ if $(X, Q)$ is locally analytically isomorphic to the quotient $\left(\mathbb{A}^{n} / \mathbb{Z}_{r}, O\right)$ under the action defined in (7.1), where $O$ denotes the image of the origin under the quotient map.

Lemma 7.2 ([13, Section 5.15]). Let $\left(a_{0}, \ldots, a_{n}\right)$ be a sequence of integers satisfying (3.1). Let $Z_{i_{1} \cdots i_{d}} \subseteq \mathbb{P}\left(a_{0}, \ldots, a_{n}\right)$ be the closed subvariety defined by

$$
X_{i_{1}}=\cdots=X_{i_{d}}=0
$$

for distinct $i_{1}, \ldots, i_{d}$. The general point of $Z_{i_{1} \ldots i_{d}}$ is locally analytically isomorphic to $(O, Q) \in \mathbb{A}^{n-d} \times Y$ where $Q \in Y$ is a singularity of type

$$
\frac{1}{h_{i_{1} \cdots i_{d}}}\left(a_{i_{1}} \ldots, a_{i_{d}}\right) \text {, }
$$

and where $h_{i_{1} \cdots i_{d}}$ is the greatest common divisor of the complement of $\left(a_{i_{1}}, \ldots, a_{i_{d}}\right)$ in $\left(a_{0}, \ldots, a_{d}\right)$. 
The next lemma is a generalization of $[13,6.10]$, where it is stated (without proof) only for complete linear systems.

Lemma 7.3 ([13, Section 6.10]). Let $\left(a_{0}, \ldots, a_{n}\right)$ be a sequence of positive integers satisfying (3.1), and let $L$ be a monomial linear system of degree $d$ on $\mathbb{P}\left(a_{0}, \ldots, a_{n}\right)$. The general member of $L$ is well-formed if and only if for all $0 \leq i<j \leq n$

(1) there exists a monomial $M \in V_{L}$ such that $M \notin\left(X_{i}, X_{j}\right)$, or

(2) we have $h_{i j}=1$ where $h_{i j}$ is as defined in the statement of Lemma 7.2.

Proof. First suppose that the general member of $L$ is well-formed and that condition 1 fails to hold for some $0 \leq i<j \leq n$. We must show that condition 2 holds. The failure of condition 1 to hold means precisely that the subvariety $Z_{i j}$ of $\mathbb{P}\left(a_{0}, \ldots, a_{n}\right)$ defined by $X_{i}=X_{j}=0$ is contained in the base locus of $L$. But if the general member of $L$ is well-formed it must be the case that the general point of $Z_{i j}$ is nonsingular. According to Lemma 7.2 we must have $h_{i j}=1$ and this is condition 2 .

Now assume that condition 1 holds for all $0 \leq i<j \leq n$. Then the codimension of the base locus of $L$ is greater than two, and it follows that the general member of $L$ is well-formed. Suppose that condition one fails to hold for some $0 \leq i<j \leq n$, but condition two does hold. Then the subvariety $Z_{i j}$ defined in the previous paragraph is in the base locus, but its general point is nonsingular. Again, it follows that the general member of $L$ is well-formed.

Proposition 7.4. Let $\left(a_{0}, \ldots, a_{3}\right)$ be a sequence of positive integers satisfy-

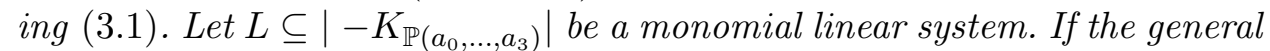
member of $L$ is quasi-smooth, then the general member of $L$ is well-formed.

Proof. Suppose that condition 1 of Lemma 7.3 fails to hold for some $0 \leq i<$ $j \leq 3$. This means that the base locus of $L$ has an irreducible component given by $X_{i}=X_{j}=0$. Let $\left\{i^{\prime}, j^{\prime}\right\}$ denote the complement of $\{i, j\}$ in the set $\{0, \ldots, 3\}$. Then according to Proposition 6.12 , there exist monomials in $V_{L}$ of the from $X_{i^{\prime}}^{p_{1}} X_{j^{\prime}}^{q_{1}} X_{e_{1}}$ and $X_{i^{\prime}}^{p_{2}} X_{j^{\prime}}^{q_{2}} X_{e_{2}}$ for distinct $e_{1}$ and $e_{2}$ such that $\left\{e_{1}, e_{2}\right\}=\{i, j\}$ In particular, this means that

$$
p_{1} a_{i^{\prime}}+p_{2} a_{j^{\prime}}+a_{e_{1}}=\sum_{l=0}^{3} a_{l} .
$$


Since we are assuming that (3.1) holds, the equation above implies that

$$
\operatorname{gcd}\left(a_{i^{\prime}}, a_{j^{\prime}}\right)=1
$$

as required to satisfy condition 2 of Lemma 7.3. Therefore, the general member of $L$ is well-formed.

Corollary 7.5. Let $\widetilde{\mathbb{P}}(\mathcal{E})$ be a weighted $\mathbb{P}\left(a_{0}, \ldots, a_{3}\right)$-bundle over $\mathbb{P}^{1}$. If the general member of $\left|-K_{\widetilde{\mathbb{P}}(\mathcal{E})}\right|$ is quasi-smooth, then it is also well-formed.

Proof. If the general member of $\left|-K_{\widetilde{\mathbb{P}}(\mathcal{E})}\right|$ is not well-formed, then the same is true for the linear system $L$ on $\mathbb{P}\left(a_{0}, \ldots, a_{3}\right)$ obtained by restricting $\left|-K_{\widetilde{\mathbb{P}}(\mathcal{E})}\right|$ to a fiber. The result now follows easily from Proposition 6.10 and Proposition 7.4.

\section{Quasi-smooth and well-formed anti-canonical hypersurfaces}

We come now to our main result, in which we classify all $\mathbb{P}\left(a_{0}, \ldots, a_{3}\right)$ bundles over $\mathbb{P}^{1}$ whose anti-canonical linear systems have a quasi-smooth member. We first check that quasi-smooth anti-canonical hypersurfaces in weighted projective bundles over $\mathbb{P}^{k}$ are necessarily Calabi-Yau varieties fibered by smaller dimensional Calabi-Yau varieties. Due to the various notions in the literature, we include here the definition of a Calabi-Yau variety that we use.

Definition 8.1 ([4, Definition 1.4.1]). Let $X$ be a normal variety that is proper over $\mathbb{C}$ and has at worst canonical singularities. We say that $X$ is a Calabi-Yau variety if $X$ has trivial dualizing sheaf and

$$
H^{1}\left(X, \mathcal{O}_{X}\right)=\cdots=H^{\operatorname{dim} X-1}\left(X, \mathcal{O}_{X}\right)=0 .
$$

In order to conclude that quasi-smooth anti-canonical hypersurfaces in weighted projective bundles are fibered by smaller dimensional Calabi-Yau varieties, we must first check that quasi-smooth anti-canonical hypersurfaces of weighted projective space are themselves Calabi-Yau varieties. 
Proposition 8.2. Let $\left(a_{0}, \ldots, a_{n}\right)$ be a sequence of integers satisfying (3.1). A quasi-smooth and well-formed member

$$
X \in\left|-K_{\mathbb{P}\left(a_{0}, \ldots, a_{n}\right)}\right|
$$

is a Calabi-Yau variety.

Proof. First we show that the dualizing sheaf $\omega_{X}$ is trivial. For any wellformed, quasi-smooth hypersurface $Z_{d}$ of degree $d$ in $\mathbb{P}\left(a_{0}, \ldots, a_{n}\right)$ we have

$$
\omega_{Z_{d}} \cong \mathcal{O}_{Z_{d}}\left(d-\sum a_{i}\right)
$$

(see [6, Theorem 3.3.4] or [13, Section 6.14]). Since $X$ is anti-canonical, it has degree $\sum a_{i}$, and it follows that $\omega_{X} \cong \mathcal{O}_{X}$. Since $X$ is quasi-smooth, it is an orbifold, and a Gorenstein orbifold necessarily has canonical singularities (see [18, Proposition 1.7]). Finally, the necessary vanishing of cohomology follows from the known cohomology of hypersurfaces in weighted projective space. (See [6, Section 3.4.3] or [13, 7.1 Lemma]).

Theorem 8.3. Let $\widetilde{\mathbb{P}}(\mathcal{E})$ be a weighted projective bundle over $\mathbb{P}^{k}$. A quasismooth and well-formed member of $\left|-K_{\widetilde{\mathbb{P}}(\mathcal{E})}\right|$ is a Calabi-Yau variety whose general fiber over $\mathbb{P}^{k}$ is a weighted Calabi-Yau hypersurface.

Proof. Let $X \in\left|-K_{\widetilde{\mathbb{P}}(\mathcal{E})}\right|$ be quasi-smooth and well-formed. Note that if $X$ is quasi-smooth, it is automatically irreducible (this follows since $X$ is transverse to a fiber). We first show that $K_{X}=0$. Let $X_{0}=\widetilde{\mathbb{P}}(\mathcal{E})_{s m} \cap X$ where $\widetilde{\mathbb{P}}(\mathcal{E})_{\text {sm }}$ denotes the non-singular locus of $\widetilde{\mathbb{P}}(\mathcal{E})$, and let $i: X_{0} \longrightarrow X$ be the inclusion map. Since $X$ is well-formed, it follows that $X_{0}$ is smooth and that the codimension of $X \backslash X_{0}$ in $X$ is at least two. The adjunction formula applied to $X_{0}$ and $\widetilde{\mathbb{P}}(\mathcal{E})_{s m}$ yields

$$
\begin{aligned}
\mathcal{O}_{X}\left(K_{X}\right) & \cong i_{*} \mathcal{O}_{X_{0}}\left(K_{X_{0}}\right) \\
& \cong i_{*}\left[\left(\mathcal{O}_{\widetilde{\mathbb{P}}(\mathcal{E})_{s m}}\left(K_{\widetilde{\mathbb{P}}(\mathcal{E})_{s m}}\right) \otimes \mathcal{O}_{\widetilde{\mathbb{P}}(\mathcal{E})_{s m}}\left(X_{0}\right) \otimes \mathcal{O}_{X_{0}}\right]\right. \\
& \cong i_{*} \mathcal{O}_{X_{0}} \\
& \cong \mathcal{O}_{X}
\end{aligned}
$$

The last isomorphism follows from the fact that both sheaves are isomorphic to the divisorial sheaf associated to the zero divisor. The dualizing sheaf of $X$ is given by $\mathcal{O}_{X}\left(K_{X}\right) \cong \mathcal{O}_{X}$, so $X$ is Gorenstein, and a Gorenstein orbifold necessarily has canonical singularities (see [18, Proposition 1.7]). 
We next show the necessary vanishing of cohomology. We use the following exact sequence.

$$
0 \longrightarrow \mathcal{O}_{\widetilde{\mathbb{P}}(\mathcal{E})}\left(K_{\widetilde{\mathbb{P}}(\mathcal{E})}\right) \longrightarrow \mathcal{O}_{\widetilde{\mathbb{P}}(\mathcal{E})} \longrightarrow \mathcal{O}_{X} \longrightarrow 0
$$

Taking the long exact sequence in cohomology, we find it suffices to show that $H^{i}\left(\widetilde{\mathbb{P}}(\mathcal{E}), \mathcal{O}_{\widetilde{\mathbb{P}}(\mathcal{E})}\right)=0$ for $1 \leq i \leq k+n-2$ and $H^{j}\left(\widetilde{\mathbb{P}}(\mathcal{E}), \mathcal{O}_{\widetilde{\mathbb{P}}(\mathcal{E})}\left(K_{\widetilde{\mathbb{P}}(\mathcal{E})}\right)\right)=0$ for $2 \leq j \leq k+n-1$. By Serre duality we have

$$
H^{j}\left(\widetilde{\mathbb{P}}(\mathcal{E}), \mathcal{O}_{\widetilde{\mathbb{P}}(\mathcal{E})}\left(K_{\widetilde{\mathbb{P}}(\mathcal{E})}\right)\right) \cong H^{k+n-j}\left(\widetilde{\mathbb{P}}(\mathcal{E}), \mathcal{O}_{\widetilde{\mathbb{P}}(\mathcal{E})}\right)^{*}
$$

and for any toric variety $Z$, we have $H^{i}\left(Z, \mathcal{O}_{Z}\right)=0$ for $i>0([5$, Corollary $7.4])$; this completes the proof that $X$ is a Calabi-Yau variety.

For the second assertion, note that generic smoothness over $\mathbb{P}^{k}$ of $q^{-1}(X)$, where $q$ is the quotient map in Definition 2.1, implies that the general fiber of $X \longrightarrow \mathbb{P}^{k}$ is quasi-smooth. Therefore, the general fiber is a well-formed anticanonical quasi-smooth hypersurface in $\mathbb{P}\left(a_{0}, \ldots, a_{n}\right)$, whence a weighted Calabi-Yau hypersurface by Proposition 8.2.

It remains to find necessary and sufficient conditions for the anti-canonical system of a weighted projective bundle to have a quasi-smooth member. We begin with a lemma that allows us to assume that the weighted sheaf from which we form the weighted projective bundle has a certain normalized form.

Lemma 8.4. Every weighted projective bundle over $\mathbb{P}^{k}$ is isomorphic to $\widetilde{\mathbb{P}}(\mathcal{E})$, where $\mathcal{E}$ is a weighted locally free sheaf of rank $n+1$ with weights $\left(a_{0}, \ldots, a_{n}\right)$ satisfying $(3.1)$ and such that

$$
\mathcal{E} \cong \bigoplus_{i=0}^{n} \mathcal{O}_{\mathbb{P}^{k}}\left(d_{i}\right)
$$

where

$$
0 \leq d_{0} \leq d_{1} \leq \cdots \leq d_{n}
$$

and such that there exists an $0 \leq i_{0} \leq n$ with

$$
d_{i_{0}}<a_{i_{0}}
$$


Proof. Let $\mathcal{E}$ be a weighted locally free sheaf with weights $\left(a_{0}, \ldots, a_{n}\right)$. Since we are only considering weighted locally free sheaves that are sums of invertible sheaves (see Definition 4.1), we may assume that

$$
\mathcal{E} \cong \bigoplus_{i=0}^{n} \mathcal{O}_{\mathbb{P}^{k}}\left(e_{i}\right)
$$

for some integers $e_{0}, \ldots, e_{n}$ and that $\mathcal{E}$ is weighted by $\left(a_{0}, \ldots, a_{n}\right)$. Given any integer $l$ we find from Lemma 4.5 that $\widetilde{\mathbb{P}}(\mathcal{E}) \cong \widetilde{\mathbb{P}}\left(\mathcal{E}^{\prime}\right)$ where

$$
\mathcal{E}^{\prime}=\bigoplus_{i=0}^{n} \mathcal{O}_{\mathbb{P}^{k}}\left(e_{i}+l a_{i}\right)
$$

Let $\lambda$ be the smallest integer such that $e_{i}+\lambda a_{i} \geq 0$ for all $0 \leq i \leq n$, define

$$
d_{i}:=e_{i}+\lambda a_{i}
$$

and renumber so that $d_{0} \leq d_{1} \leq \cdots \leq d_{n}$. If $d_{i} \geq a_{i}$ for all $0 \leq i \leq n$, then we have $e_{i}+(\lambda-1) a_{i} \geq 0$, but this contradicts the minimality of the integer $\lambda$. Therefore $d_{0}, \ldots, d_{n}$ give the required integers.

We are now in position to prove our classification result for anti-canonical systems on $\mathbb{P}\left(a_{0}, \ldots, a_{3}\right)$-bundles over $\mathbb{P}^{1}$.

Theorem 8.5. Let $\left(d_{0}, \ldots, d_{3}\right)$ be a sequence of integers satisfying (8.2) and (8.3), and let $\left(a_{0}, \ldots, a_{3}\right)$ be a sequence of positive integers satisfying (3.1). Let $\mathcal{E}$ be the weighted locally free sheaf

$$
\mathcal{E}:=\mathcal{O}_{\mathbb{P}^{1}}\left(d_{0}\right) \oplus \cdots \oplus \mathcal{O}_{\mathbb{P}^{1}}\left(d_{3}\right)
$$

with weights $\left(a_{0}, \ldots, a_{3}\right)$. Let $L$ denote the linear system on $\mathbb{P}\left(a_{0}, \ldots, a_{3}\right)$ obtained by restricting $\left|-K_{\widetilde{\mathbb{P}}(\mathcal{E})}\right|$ to a fiber, let $V_{L}$ be its associated vector space of homogeneous forms, and let $\eta$ be defined via (6.3). Then the linear system $\left|-K_{\widetilde{\mathbb{P}}(\mathcal{E})}\right|$ has a well-formed quasi-smooth member if and only if the following conditions hold.

(1) The general member of the linear system $L$ is quasi-smooth.

(2) For all $0 \leq i \leq 3$

(a) there exists a monomial of the form $X_{i}^{p}$ in $V_{L}$, or

(b) there exists an integer $j \neq i$ and a monomial $M \in V_{L}$ of the form $X_{j} X_{i}^{p}$ such that $\eta(M)=0$, or 
(c) there exist distinct integers $j_{1} \neq i$ and $j_{2} \neq i$ and two monomials $M_{1}$ and $M_{2}$ of the form $X_{j_{1}} X_{i}^{p}$ and $X_{j_{2}} X_{i}^{p}$ respectively such that $\eta\left(M_{1}\right)>0$ and $\eta\left(M_{2}\right)>0$.

(3) For all $0 \leq i<j \leq 3$

(a) there exists a monomial of the form $X_{i}^{p} X_{j}^{q} \in V_{L}$ or

(b) there exist monomials of the form

$$
N_{1}=X_{i}^{p_{1}} X_{j}^{q_{1}} X_{e_{1}} \in V_{L}
$$

and

$$
N_{2}=X_{i}^{p_{2}} X_{j}^{q_{2}} X_{e_{2}} \in V_{L}
$$

in $V_{L}$ such that $e_{1}$ and $e_{2}$ are distinct and such that

$$
\begin{aligned}
\left(\left\lceil\frac{p_{1}}{a_{j}}\right\rceil+\left\lceil\frac{q_{1}}{a_{i}}\right\rceil\right) & \eta\left(N_{2}\right)+\left(\left\lceil\frac{p_{2}}{a_{j}}\right\rceil+\left\lceil\frac{q_{2}}{a_{i}}\right\rceil\right) \eta\left(N_{1}\right) \\
& +\left(\left\lceil\frac{p_{1}}{a_{j}}\right\rceil\left\lceil\frac{p_{2}}{a_{j}}\right\rceil+\left\lceil\frac{q_{1}}{a_{i}}\right\rceil\left\lceil\frac{q_{2}}{a_{i}}\right\rceil\right)\left(a_{i} d_{j}-a_{j} d_{i}\right)=0 .
\end{aligned}
$$

Proof. Recall that if the general member of $\left|-K_{\widetilde{\mathbb{P}}(\mathcal{E})}\right|$ is quasi-smooth, then it is automatically well-formed by Corollary 7.5. Now suppose that the general member of $\left|-K_{\widetilde{\mathbb{P}}(\mathcal{E})}\right|$ is quasi-smooth. From Proposition 6.10 we see that condition 1 holds. We must show that conditions 2 and 3 hold. We proceed according to the codimension of the base locus of $\left|-K_{\widetilde{\mathbb{P}}(\mathcal{E})}\right|$. The equations that define the base locus are given by monomials in the variables $X_{0}, \ldots, X_{3}$. If the base locus is empty, then conditions 2 (a) and 3 (a) hold. Also, there can be no fixed component because then $L$ would have a fixed component, and this would contradict the fact that condition 1 holds. This leaves two possibilities: either the base locus of $\left|-K_{\widetilde{\mathbb{P}}(\mathcal{E})}\right|$ has codimension three or it has codimension two.

We first consider the case in which

$$
\operatorname{codim} \operatorname{Bs}\left|-K_{\widetilde{\mathbb{P}}(\mathcal{E})}\right|=3
$$

and we suppose that $C$ is a component of the base locus given by

$$
X_{j_{1}}=X_{j_{2}}=X_{j_{3}}=0
$$

for

$$
0 \leq j_{1}<j_{2}<j_{3} \leq 3
$$


Let $F$ denote the equation for a general member of $\left|-K_{\widetilde{\mathbb{P}}(\mathcal{E})}\right|$. Since $Z(F)$ is quasi-smooth we find, in particular, that the system of equations

$$
\frac{\partial F}{\partial X_{j_{1}}}=\frac{\partial F}{\partial X_{j_{2}}}=\frac{\partial F}{\partial X_{j_{3}}}=0
$$

has no non-trivial solution on $C$. Restricting to $C$, the equations in (8.5) become

$$
\phi_{j_{1}} X_{i}^{p_{1}}=\phi_{j_{2}} X_{i}^{p_{2}}=\phi_{j_{3}} X_{i}^{p_{3}}=0
$$

for $i \notin\left\{j_{1}, j_{2}, j_{3}\right\}$ and for forms

$$
\phi_{j_{l}} \in H^{0}\left(\mathbb{P}^{1}, \mathcal{O}_{\mathbb{P}^{1}}\left(\eta\left(X_{j_{l}} X_{i}^{p_{l}}\right)\right)\right)
$$

At least one of the forms must be non-zero otherwise there could be no quasi-smooth member. If only one form is non-zero, it must have degree zero, otherwise it would have a zero. This is condition 2 (b). If at least two of the $\phi_{j l}$ are non-zero, and neither has degree zero, then condition 2 (c) holds. Finally, since the dimension of the base locus is zero, it follows that condition 3 (a) holds.

Now assume that

$$
\operatorname{codim} \operatorname{Bs}\left|-K_{\widetilde{\mathbb{P}}(\mathcal{E})}\right|=2 .
$$

If the base locus has a component of dimension zero, then we are in the situation of the previous paragraph. Let $C$ be a component of the base locus given by $X_{e_{1}}=X_{e_{2}}=0$ and let $\{i, j\}$ denote the complement of $\left\{e_{1}, e_{2}\right\}$ in $\{0,1,2,3\}$. Observe that

$$
C \cong \widetilde{\mathbb{P}}\left(\mathcal{O}_{\mathbb{P}^{1}}\left(d_{i}\right) \oplus \mathcal{O}_{\mathbb{P}^{1}}\left(d_{j}\right)\right)
$$

with weights $\left(a_{i}, a_{j}\right)$. Furthermore, we claim that $\operatorname{gcd}\left(a_{i}, a_{j}\right)=1$. Indeed, since condition one is satisfied, Proposition 6.12 ensures the existence of a monomial of the form $X_{i}^{p_{1}} X_{j}^{q_{1}} X_{e_{1}}$. Hence we have

$$
p_{1} a_{i}+p_{2} a_{j}+a_{e_{1}}=\sum a_{i}
$$

from which the claim follows since the weights $\left(a_{0}, \ldots, a_{3}\right)$ satisfy $(3.1)$. Therefore, it follows from Lemma 5.2 that

$$
C \cong \mathbb{P}\left(\mathcal{O}_{\mathbb{P}^{1}}\left(a_{j} d_{i}\right) \oplus \mathcal{O}_{\mathbb{P}^{1}}\left(a_{i} d_{j}\right)\right)
$$


Arguing as before we find that the quasi-smoothness of the general member $Z(F)$ implies that the system of equations

$$
\frac{\partial F}{\partial X_{e_{1}}}=\frac{\partial F}{\partial X_{e_{2}}}=0
$$

has no non-trivial solution on $C$. If we restrict the partial derivatives (8.7) to $C$ we obtain the equation for two divisors

$$
D_{e_{1}}:=Z\left(\left.\frac{\partial F}{\partial X_{e_{1}}}\right|_{C}\right) \quad \text { and } \quad D_{e_{2}}:=Z\left(\left.\frac{\partial F}{\partial X_{e_{2}}}\right|_{C}\right)
$$

on $C$ that meet transversally. Therefore, the existence of a non-trivial solution on $C$ to (8.7) is equivalent to the intersection number $D_{e_{1}} \cdot D_{e_{2}}$ not vanishing. Furthermore, since condition 1 is satisfied, Proposition 6.12 guarantees the existence of monomials of the form $N_{1}$ and $N_{2}$ in $V_{L}$, and we must check that (8.4) is satisfied. The presence of the monomials $N_{1}$ and $N_{2}$ in $V_{L}$ allows us to use Lemma 5.2 to determine the type of the two divisors on $C$. Referring to Example 5.1 for notation and terminology, we find that

$$
D_{e_{1}} \text { has type }\left(\left\lceil\frac{p_{1}}{a_{j}}\right\rceil+\left\lceil\frac{q_{1}}{a_{i}}\right\rceil, \eta\left(N_{1}\right)-\left\lceil\frac{p_{1}}{a_{j}}\right\rceil a_{j} d_{i}-\left\lceil\frac{q_{1}}{a_{i}}\right\rceil a_{i} d_{j}\right)
$$

and

$$
D_{e_{2}} \text { has type }\left(\left\lceil\frac{p_{2}}{a_{j}}\right\rceil+\left\lceil\frac{q_{2}}{a_{i}}\right\rceil, \eta\left(N_{2}\right)-\left\lceil\frac{p_{2}}{a_{j}}\right\rceil a_{j} d_{i}-\left\lceil\frac{q_{2}}{a_{i}}\right\rceil a_{i} d_{j}\right),
$$

and the intersection number $D_{e_{1}} \cdot D_{e_{2}}$ simplifies to the left-hand side of (8.4).

Now suppose that conditions 1-3 hold. We will show that the general member is quasi-smooth. It suffices to proceed according to irreducible components present in the base locus of $\left|-K_{\widetilde{\mathbb{P}}(\mathcal{E})}\right|$. If the base locus is empty, then Proposition 6.7 guarantees the existence of a quasi-smooth member. Condition 1 prevents there from being a fixed component in the base locus. Now suppose that the base locus has an irreducible component $C$ of codimension 2 given by $\left\{X_{e_{1}}=X_{e_{2}}=0\right\}$. Then we must check that it cannot be the case that all the members fail to be quasi-smooth on $C$. As described in the previous paragraph, condition 3 (b) prevents this from happening. Note that we also need condition 1 to ensure that the two divisors $D_{e_{1}}$ and $D_{e_{2}}$ from the previous paragraph intersect transversally. It remains to check the case in which the base locus has an irreducible component $C$ of codimension three. Again, as described in the previous paragraph, conditions 2 (b) and 
2 (c) ensure that not every member fails to be quasi-smooth on $C$. This completes the proof.

Corollary 8.6. With the notation and assumptions of Theorem 8.5 the general member of the linear system $\left|-K_{\widetilde{\mathbb{P}}(\mathcal{E})}\right|$ is quasi-smooth and wellformed if and only if the following conditions hold.

(1) For all $0 \leq i \leq 3$ there exists a monomial in $V_{L}$ that does not involve $X_{i}$.

(2) For all $0 \leq i \leq 3$

(a) there exists a monomial of the form $X_{i}^{p}$ in $V_{L}$, or

(b) there exists an integer $j \neq i$ and a monomial $M \in V_{L}$ of the form $X_{j} X_{i}^{p}$ such that $\eta(M)=0$, or

(c) there exist distinct integers $j_{1} \neq i$ and $j_{2} \neq i$ and two monomials $M_{1}$ and $M_{2}$ of the form $X_{j_{1}} X_{i}^{p}$ and $X_{j_{2}} X_{i}^{p}$ respectively such that $\eta\left(M_{1}\right)>0$ and $\eta\left(M_{2}\right)>0$.

(3) For all $0 \leq i<j \leq 3$

(a) there exists a monomial of the form $X_{i}^{p} X_{j}^{q} \in V_{L}$ or

(b) there exist monomials of the form

$$
N_{1}=X_{i}^{p_{1}} X_{j}^{q_{1}} X_{e_{1}} \in V_{L}
$$

and

$$
N_{2}=X_{i}^{p_{2}} X_{j}^{q_{2}} X_{e_{2}} \in V_{L}
$$

in $V_{L}$ such that $e_{1}$ and $e_{2}$ are distinct and such that

$$
\begin{aligned}
\left(\left\lceil\frac{p_{1}}{a_{j}}\right\rceil+\left\lceil\frac{q_{1}}{a_{i}}\right\rceil\right) & \eta\left(N_{2}\right)+\left(\left\lceil\frac{p_{2}}{a_{j}}\right\rceil+\left\lceil\frac{q_{2}}{a_{i}}\right\rceil\right) \eta\left(N_{1}\right) \\
& +\left(\left\lceil\frac{p_{1}}{a_{j}}\right\rceil\left\lceil\frac{p_{2}}{a_{j}}\right\rceil+\left\lceil\frac{q_{1}}{a_{i}}\right\rceil\left\lceil\frac{q_{2}}{a_{i}}\right\rceil\right)\left(a_{i} d_{j}-a_{j} d_{i}\right)=0
\end{aligned}
$$

Proof. This follows immediately from Theorem 8.5 and Proposition 6.12.

\section{Elliptically fibered Calabi-Yau threefolds}

Using techniques similar to those used in the proof of Theorem 8.5 we can prove the analogous result for the case of Calabi-Yau 3-folds fibered over $\mathbb{P}^{2}$ whose general fiber is a genus one curve in $\mathbb{P}(1,1,1), \mathbb{P}(1,1,2)$, or $\mathbb{P}(1,2,3)$. The statement of the full result follows. It turns out that these Calabi-Yau 3 -folds are non-singular. See [17, Section 4.4] for details. 
Theorem 9.1. Let $\left(d_{0}, d_{1}, d_{2}\right)$ be a sequence of integers that satisfy (8.2) and (8.3), and let $\left(a_{0}, a_{1}, a_{2}\right)$ be a sequence of positive integers satisfying (3.1). Let $\mathcal{E}$ be the weighted locally free sheaf

$$
\mathcal{E}:=\mathcal{O}_{\mathbb{P}^{2}}\left(d_{0}\right) \oplus \mathcal{O}_{\mathbb{P}^{2}}\left(d_{1}\right) \oplus \mathcal{O}_{\mathbb{P}^{2}}\left(d_{3}\right)
$$

with weights $\left(a_{0}, a_{1}, a_{2}\right)$. Let $L$ denote the linear system on $\mathbb{P}\left(a_{0}, a_{1}, a_{2}\right)$ obtained by restricting $\left|-K_{\widetilde{\mathbb{P}}(\mathcal{E})}\right|$ to a fiber, and let $\eta$ be defined via (6.3) Then the linear system $\left|-K_{\widetilde{\mathbb{P}}(\mathcal{E})}\right|$ has a well-formed non-singular member if and only if the following conditions hold.

(1) For all $i \in\{0,1,2\}$ there exists a monomial in $V_{L}$ that does not involve $X_{i}$.

(2) For all $i \in\{0,1,2\}$ either

(a) there exists a monomial of the form $X_{i}^{p}$ in $V_{L}$ or

(b) there exists an integer $j \neq i$ and a monomial $M$ of the form $X_{i}^{p} X_{j}$ such that $\eta(M)=0$.

There are 92 families of elliptically fibered Calabi-Yau 3-folds over $\mathbb{P}^{2}$ arising as members of the anti-canonical linear system of $\mathbb{P}\left(a_{0}, a_{1}, a_{2}\right)$-bundle over $\mathbb{P}^{2}$. We list these families in the appendix.

\section{Final remarks}

It is natural to ask to what extent the Calabi-Yau 3-folds we have found are new. From the point of view of mirror symmetry, they are not new in the sense that they can be related to Batyrev's mirror symmetry construction (see [1]). Although the varieties $\widetilde{\mathbb{P}}(\mathcal{E})$ are not, in general, reflexive (i.e., Fano and Gorenstein), their Calabi-Yau hypersurfaces have crepant resolutions that are anti-canonical hypersurfaces in reflexive toric varieties, and these have all been classified in [15]. The existence of these resolutions is established in [17] by checking that the Newton polyhedra of all toric weighted projective bundles admitting quasi-smooth anti-canonical hypersurfaces are reflexive.

The varieties are interesting, however, for other reasons. For example, in [16] fiber structures of toric Calabi-Yau hypersurfaces are considered in the reflexive case. It can be tricky to find fiber structures from that point of view, and as far as the author is aware, no such explicit description of weighted K3-fibered Calabi-Yau 3-folds as given here has appeared before, and it was not necessary to have reflexive toric varieties to write them down. 
Additionally, our result gives a direct generalization of Reid's discovery of the "famous 95" families of weighted K3 hypersurfaces, and is thus interesting from the point of view of explicit birational geometry.

\section{Appendix A. Some data in the K3 case}

We give here a partial list of K3-fibered Calabi-Yau hypersurfaces in toric weighted projective bundles over $\mathbb{P}^{1}$. The list is in the form of a hash table in Macaulay 2 ([9]). (Note: The raw Macaulay 2 output has been altered by inserting line-breaks.) Each entry in the hash table consists of a key and an ASCII arrow "=>" followed by a value. Each key is a list of four integers, and each value is a list of lists of integers. If $\{a, b, c, d\}$ is a key and $\{e, f, g, h\}$ is a member of the list of values for $\{a, b, c, d\}$, then the general member of the linear system $\left|-K_{\widetilde{\mathbb{P}}(\mathcal{E})}\right|$ is quasi-smooth where $\mathcal{E}$ is the weighted locally free sheaf

$$
\mathcal{O}_{\mathbb{P}^{1}}(e) \oplus \mathcal{O}_{\mathbb{P}^{1}}(f) \oplus \mathcal{O}_{\mathbb{P}^{1}}(g) \oplus \mathcal{O}_{\mathbb{P}^{1}}(h)
$$

weighted by $(a, b, c, d)$. The complete list of all 3723 families and details of the calculation appear in [17].

$$
\begin{aligned}
\{1,1,1,1\} & = \\
& \{\{0,0,0,0\},\{0,0,0,1\},\{0,0,0,2\},\{0,0,1,1\}, \\
& \{0,0,2,2\},\{0,1,1,1\},\{0,1,1,2\},\{0,1,1,3\}, \\
& \{0,1,1,4\}\} \\
\{1,1,1,2\} & => \\
& \{\{0,0,0,0\},\{0,0,0,1\},\{0,0,0,2\},\{0,0,1,1\}, \\
& \{0,0,2,2\},\{0,1,1,1\},\{0,1,1,2\},\{0,1,1,3\}, \\
& \{0,1,1,4\},\{0,1,1,5\},\{1,1,1,1\},\{0,0,1,0\}, \\
& \{0,0,2,0\},\{0,1,2,1\},\{0,1,3,1\},\{1,1,2,1\}, \\
& \{0,1,1,0\},\{0,2,2,0\},\{1,2,2,1\},\{1,2,3,1\}, \\
& \{1,2,4,1\},\{1,0,0,0\},\{2,0,0,0\},\{1,0,1,0\}, \\
& \{2,0,2,0\},\{1,1,1,0\},\{2,1,1,0\},\{3,1,1,0\}, \\
& \{4,1,1,0\},\{5,1,1,0\},\{2,1,1,1\},\{2,1,2,1\}, \\
& \{3,1,2,1\}\} \\
& => \\
& \{\{0,0,0,0\},\{0,0,0,1\},\{0,0,0,2\},\{0,0,1,1\}, \\
& \{0,0,2,2\},\{0,1,1,1\},\{0,1,1,2\},\{0,1,1,3\}, \\
& \{0,1,1,4\},\{0,1,1,5\},\{0,1,1,6\},\{1,1,1,1\}, \\
& \{1,1,1,2\},\{1,1,2,2\},\{0,0,1,0\},\{0,0,2,0\}, \\
& \{0,1,2,1\},\{0,1,1,0\},\{1,0,0,0\},\{2,0,0,0\}, \\
& \{1,0,1,0\}\}
\end{aligned}
$$




$$
\begin{aligned}
& \{1,1,2,2\} \Rightarrow \\
& \{\{0,0,0,0\},\{0,0,0,1\},\{0,0,0,2\},\{0,0,1,1\} \text {, } \\
& \{0,0,2,2\},\{0,1,1,1\},\{0,1,1,2\},\{0,1,1,3\} \text {, } \\
& \{1,1,1,1\},\{1,1,1,2\},\{0,1,0,1\},\{0,2,0,2\} \text {, } \\
& \{1,2,1,2\},\{1,2,1,3\},\{1,2,1,4\},\{1,2,1,5\} \text {, } \\
& \{0,1,0,0\},\{0,2,0,0\},\{0,2,1,1\},\{0,3,1,1\} \text {, } \\
& \{1,2,1,1\},\{1,0,0,0\},\{2,0,0,0\},\{1,0,0,1\} \text {, } \\
& \{2,0,0,2\},\{1,1,0,1\},\{2,1,1,1\},\{2,1,1,2\} \text {, } \\
& \{1,1,0,0\},\{0,0,1,0\},\{0,0,2,0\},\{1,0,1,0\} \text {, } \\
& \{2,0,2,0\},\{1,1,1,0\},\{1,1,2,0\},\{1,1,3,0\} \text {, } \\
& \{1,1,4,0\},\{1,1,5,0\},\{1,1,6,0\},\{1,1,2,1\} \text {, } \\
& \{2,1,2,1\},\{2,1,3,1\},\{2,1,4,1\}\} \\
& \{1,1,2,3\}=> \\
& \{\{0,0,0,0\},\{0,0,0,1\},\{0,0,0,2\},\{0,0,1,1\} \text {, } \\
& \{0,0,2,2\},\{0,1,1,1\},\{0,1,1,2\},\{0,1,1,3\} \text {, } \\
& \{0,1,1,4\},\{1,1,1,1\},\{1,1,1,2\},\{1,1,1,3\} \text {, } \\
& \{1,1,2,2\},\{1,2,2,2\},\{2,2,2,2\},\{0,0,1,0\} \text {, } \\
& \{0,0,2,0\},\{0,1,2,1\},\{0,1,3,1\},\{1,1,2,1\} \text {, } \\
& \{1,1,3,2\},\{1,2,3,2\},\{0,1,0,1\},\{0,2,0,2\} \text {, } \\
& \{1,2,1,2\},\{1,2,1,3\},\{1,2,1,4\},\{1,2,1,5\} \text {, } \\
& \{1,2,1,6\},\{1,3,1,3\},\{0,1,0,0\},\{0,2,0,0\} \text {, } \\
& \{0,2,1,1\},\{1,2,1,1\},\{1,4,1,3\},\{2,3,2,2\} \text {, } \\
& \{2,4,2,2\},\{0,1,1,0\},\{0,2,2,0\},\{1,2,2,1\} \text {, } \\
& \{1,3,3,2\},\{1,3,2,1\},\{1,4,2,1\},\{1,5,2,1\} \text {, } \\
& \{1,4,3,2\},\{1,0,0,0\},\{2,0,0,0\},\{1,0,0,1\} \text {, } \\
& \{2,0,0,2\},\{1,1,0,1\},\{2,1,0,1\},\{3,1,0,1\} \text {, } \\
& \{2,1,1,1\},\{2,1,1,2\},\{3,1,1,3\},\{4,1,1,3\} \text {, } \\
& \{2,2,1,2\},\{3,2,1,2\},\{3,2,2,2\},\{4,2,2,2\} \text {, } \\
& \{1,1,0,0\},\{2,2,0,0\},\{3,2,1,1\},\{1,0,1,0\} \text {, } \\
& \{2,0,2,0\},\{1,1,1,0\},\{2,1,1,0\},\{3,1,1,0\} \text {, } \\
& \{4,1,1,0\},\{2,1,2,1\},\{3,1,2,1\},\{4,1,2,1\} \text {, } \\
& \{5,1,2,1\},\{1,1,0,2\},\{1,1,0,3\},\{1,1,0,4\} \text {, } \\
& \{1,1,0,5\},\{1,1,0,6\},\{1,1,0,7\},\{2,1,1,3\} \text {, } \\
& \{2,1,1,4\},\{2,1,1,5\}\}
\end{aligned}
$$

\section{Appendix B. Data in the elliptic case}

In this section we give the complete list of the elliptically fibered CalabiYau 3-folds discussed in Section 9. This list is to be interpreted in a manner similar to the list in Appendix A.

$$
\begin{aligned}
\{1,1,1\} & \Rightarrow \\
& \{\{0,0,0\},\{0,0,1\},\{0,0,2\},\{0,0,3\},\{0,1,1\}, \\
& \{0,1,2\},\{0,1,3\},\{0,2,3\}\}
\end{aligned}
$$




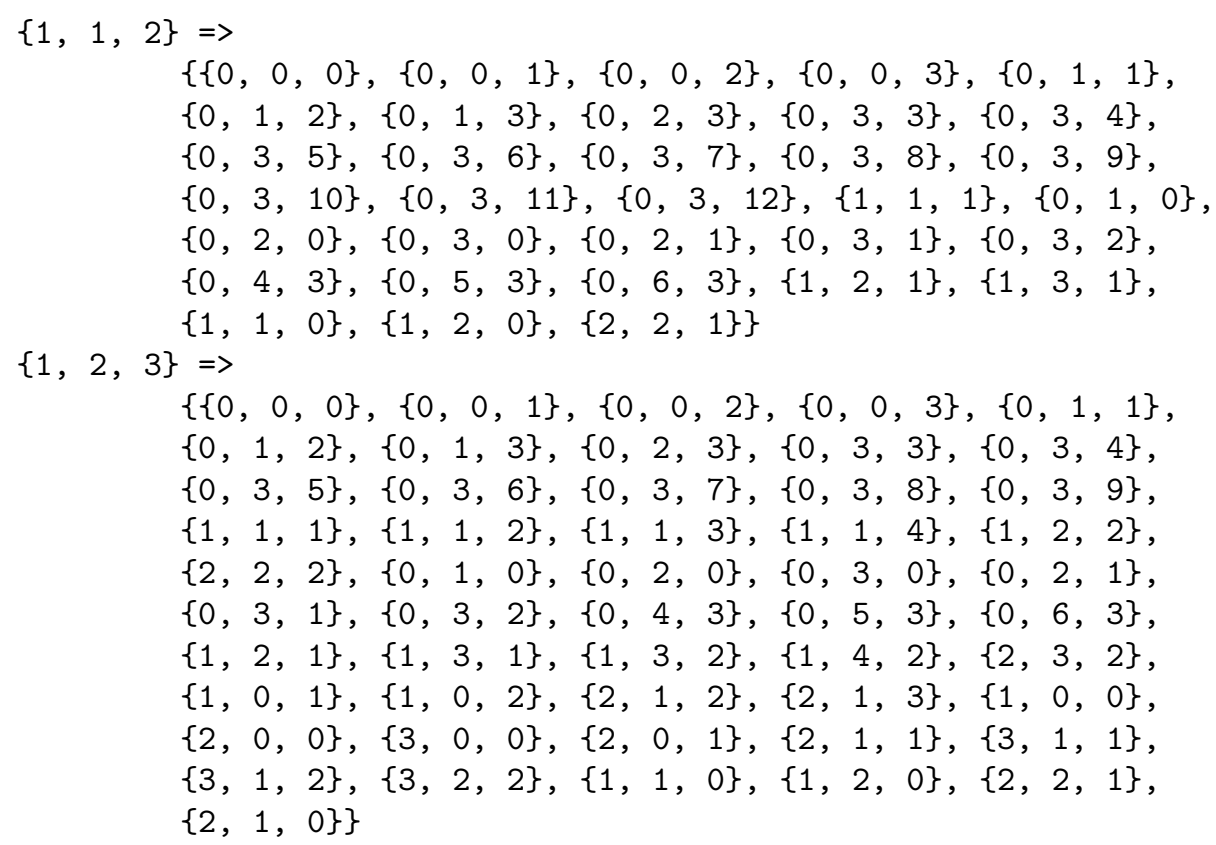

\section{References}

[1] V.V. Batyrev, Dual polyhedra and mirror symmetry for Calabi-Yau hypersurfaces in toric varieties, J. Algebr. Geom. 3(3) (1994), 493-535.

[2] A. Corti and M. Reid (eds.), Explicit birational geometry of 3-folds, London Mathematical Society Lecture Note Series, vol. 281, Cambridge University Press, Cambridge, 2000.

[3] D. Cox, The homogeneous coordinate ring of a toric variety, J. Algebr. Geom. 4 (1995), 17-50.

[4] D.A. Cox and S. Katz, Mirror symmetry and algebraic geometry, in Mathematical Surveys and Monographs, 68, American Mathematical Society, Providence, RI, 1999.

[5] V.I. Danilov, The geometry of toric varieties, Russ. Math Surv. 33 (1978), 97-154.

[6] I. Dolgachev, Weighted projective varieties, in Group Actions and Vector Fields (Vancouver, B.C., 1981), Lecture Notes in Math. 956, Springer, Berlin, 1982, 34-71.

[7] W. Fulton, Introduction to toric varieties, in Annals of Math. Stud. 131, Princeton University Press, Princeton, 1993. 
[8] W. Fulton, Intersection theory, 2nd ed., in Ergebnisse der Mathematik und ihrer Grenzgebiete. 3. Folge. A Series of Modern Surveys in Mathematics, 2, Springer-Verlag, Berlin, 1998.

[9] D.R. Grayson and M.E. Stillman, Macaulay 2, a software system for research in algebraic geometry, available at: http://www.math.uiuc. edu/Macaulay2/.

[10] P. Griffiths and J. Harris, Principles of algebraic geometry, Wiley Classics Library, John Wiley \& Sons, Inc., New York, 1994.

[11] A. Grothendieck, Éléments de géométrie algébrique. II. Étude globale élémentaire de quelques classes de morphismes, Inst. Hautes Études Sci. Publ. Math. (8) (1961).

[12] R. Hartshorne, Algebraic geometry, in Graduate Texts in Mathematics, 52, Springer-Verlag, New York, 1977.

[13] A.R. Iano-Fletcher, Working with weighted complete intersections, in 'Explicit Birational Geometry of 3-Folds' eds. A. Corti and M. Reid, London Mathematical Society Lecture Note Series, 281, Cambridge University Press, Cambridge, 2000, 101-173.

[14] M. Kobayashi, private communication.

[15] M. Kreuzer and H. Skarke, Complete classification of reflexive polyhedra in four dimensions, Adv. Theor. Math. Phys. 4(6) (2000), 1209-1230.

[16] Reflexive polyhedra, weights and toric Calabi-Yau fibrations, Rev. Math. Phys. 14(4) (2002), 343-374.

[17] J.P. Mullet, Fibered Calabi-Yau varieties and toric varieties, $\mathrm{PhD}$ thesis, University of Illinois at Urbana-Champaign, 2006.

[18] M. Reid, Canonical 3-folds, in 'Journées de géométrie algébrique d'Angers', ed. A. Beauville, Sijthoff \& Nordhoff, Alphen aan den Rijn, 1980, 273-310.

Department of Mathematics

The Ohio State University

Columbus

OH 43210

USA

E-mail address: mullet@math.ohio-state.edu

RECEIVED FEBRUARY 23, 2008 